\title{
BEHAVIOR OF PLANTS IN UNVENTILATED CHAMBERS ${ }^{1}$
}

\section{F. C. Newcombe and Etta A. Bowerman}

Within the past five years the writers have heard several botanists of good standing express their belief in the need of ventilation for plants, the notion being that plants in small chambers, in dark rooms, and even fungi in closed vessels, would grow and react better if the surrounding air was in motion.

This notion probably arose from the vagueness which, up to the last decade, existed as to the cause of "bad air" in rooms occupied by human beings, and the discovery, about ten years ago, that the ill effects of bad air could be removed by setting the air in motion. For over thirty years it has been known that bad air in unventilated rooms is not due to the accumulation of carbon dioxide. Various conjectures were offered as solutions of the problem. Perhaps it is not remarkable that some botanists should have imagined that their plants needed moving air for their well-being. This assumption of the need of moving air did not concern at all the oxygen or the carbon dioxide supply, but something else not more precisely defined.

Since, within the last decade, bad air in a room has been found in human hygiene to be due to excessive temperature and humidity, as the matter has been summarized by Hill, Flack, Rowlands, and Walker, ${ }^{2}$ the effect of these conditions being manifested in the enlargement of the peripheral blood vessels and the consequent derangement in the heart-beat and the respiratory rhythm, it would seem difficult to apply the same causes to plants which have no circulation corresponding to that of the higher animals. Moreover, plants in a confined space almost never raise the temperature to the optimum for their growth; and, although they make the atmosphere very humid, humidity is generally favorable to their growth. But however improbable it may seem theoretically that experimental plants in confined spaces require ventilation, the plant physiologist needs to be assured

${ }^{1}$ Publication No. 168 from the Botanical Department of the University of Michigan.

2 Hill, Flack, Rowlands, and Walker. Influence of Atmosphere on Health. Smithson. Misc. Coll. 60: 1. I913. 
by exact experiment. A narrative of such experimentation is given in the following pages.

\section{EXPERIMENTATION}

\section{Growth of Plants in Unventilated Small Chambers}

Series 1 .- -The test for this series was made with plants confined under glass bell jars or under zinc cylinders closed at one end, bell jars and zinc cylinders having a capacity of 6 to 5 liters each, according to the size of the plants to be covered, and all placed in a darkroom of about 12 cubic meters capacity. For the standard, an equal number of plants of the same species were grown in uncovered pots in the same darkroom. An electric blower was employed to send day and night the air of the dark room in a gentle current over both sets of preparations. The temperature over the uncovered plants varied from $21^{\circ}$ to $24^{\circ} \mathrm{C}$.; in the bell jars and metal cylinders it was usually one degree higher by the thermometer, the difference in the showing of the thermometer being due probably to the current of air. The humidity over the uncovered seedlings varied from 35 percent to 58 percent; under the covers it stood near Ioo percent.

The most of the work was done with seedlings, as seedlings are usually the plants employed for darkroom work. The seeds were planted in sphagnum moss in pots, and, as soon as the seedlings appeared above the moss, half of the pots were placed under the covers while the other half were exposed to the air current.

Observations and records were made every day through the period of the tests which varied with different sets from 5 to 17 days. The points for which observations were made were size of plants, time of unfolding of leaves, size of leaves, time of falling over of the seedlings, and general appearance.

The species and varieties of seedlings employed, with the numbers of plants, were as follows: Zea mais L. 140, Zea mais var. everta Sturtev. 54, Raphanus sativus L. 3I, Fagopyrum esculentum Moench. 25, Pisum sativum L. 65, Lupinus albus L. 19.

Observation and measurements showed but slight differences between the plants raised under the two conditions. Members of the laboratory staff were asked to tell the differences between the two sets of plants when the pots were placed in two groups on a table, but were never able to name distinguishing features unless the comparison was made at a time near that of the exhaustion of the seedlings. 
At such a time the seedlings from the confined air showed earlier exhaustion by falling over.

All species grow a little faster and reach their condition of exhaustion one to two days earlier in the small chambers. Measurements showed the Fagopyrum and the Lupinus attaining a slightly greater height in the current of air, while Zea, Raphanus, and Pisum grew higher under the bell jars. Only the Zea and Pisum produce, in the darkroom, leaves above the cotyledons. Measurements indicated slightly larger leaves from the quiet confined air.

Series II.-This series of tests was made in another darkroom of the same size as the preceding, and bell jars and zinc cylinders were used as before for one set of plants. Intermingled with the bell jars and cylinders stood the uncovered control plants, and a current of air, brought in from the adjoining greenhouse by a small blower and returned to the greenhouse, kept a constant draft around all the preparations. Except for the cooling effect of the greater transpiration from the exposed plants and the pots, the covered and uncovered plants must have been at the same temperature.

In general the seedlings used were planted in pots of earth, allowed to stand in the greenhouse till breaking through the ground, then placed under experiment in the darkroom. Some tests were made with seedlings which had reached a height of 10 to $20 \mathrm{~cm}$. in the greenhouse before being placed in the darkroom. Since these latter showed in no way a behavior different from that of the younger seedlings, except that they did not live as long, they will not be given special consideration in the record.

A very thorough test was made with Zea mais L. (yellow dent), of which more than 400 seedlings were used in 7 distinct trials. The record for one of these trials with Zea is given here as representative for all:

"July 2, 1917. 30 seedlings about $10 \mathrm{~cm}$. above earth in pots placed under zinc cylinders. 26 similar seedlings uncovered.

"July 9. All seedlings in each set have fallen over, none dead. Plants under cover, taller, thicker, and better looking than the uncovered. The ro largest plants of the covered set average $47 \mathrm{~cm}$. from ground to tip of longest leaf; Io largest uncovered plants average $39 \mathrm{~cm}$. Of covered plants, $\mathrm{I}_{3}$ have 3 leaves each; of uncovered, 7 plants have 3 leaves each. Many roots growing up into air from earth in covered pots; none in uncovered pots. Temperature has varied from $20^{\circ}$ to $25^{\circ}$."

Besides the Zea of the last-described experiment, there were tested 
by the same means 23 seedlings of Pisum sativum L., 26 seedlings of Phaseolus vulgaris L. (white kidney bean), I5 seedlings of Phaseolus vulgaris L. (black kidney bean), 55 seedlings of Brassica alba (L.) Boiss. Duration of the experiments was from 4 to Io days.

The seedlings of Pisum and Phaseolus showed a greater height in the covered pots than in the uncovered, and, on the average, slightly larger leaves in the covered. The Brassica showed no differences under the two conditions, height of plants and size of cotyledons averaging the same. All species showed falling over about one day earlier in the covered pots. In the last days of some of the experiments some pots of seedlings under cover were attacked by damping off; the "falling over" mentioned is due to exhaustion of the plants, not to damping off.

Three species of larger leafy plants were subjected to the same test as the foregoing seedlings. The air in the darkroom being constantly brought in from the greenhouse in which the potted plants had been growing, there was no danger of the transposed plants suffering from a too dry atmosphere. The Ricinus plants used and the Bryophyllum were from 50 to $65 \mathrm{~cm}$. tall, and cylinders correspondingly high were used to cover them. The experiments were made in July and August.

There were used 3 plants of Ricinus communis L. under zinc cylinders, and 6 plants uncovered. No differences in their growth or other behavior could be detected during the Io days of trial. All began dropping their leaves after 7 days, and in Io days from the beginning of the experiment all leaves had fallen.

Twelve plants of Bryophyllum calycinum Salisb. were put under experiment, 6 for 16 days and 6 for 27 days; in each experiment, 3 plants were kept under zinc cylinders and 3 were left uncovered and thus exposed to the moving air. No differences in behavior could be seen. The new leaves unfolded in the dark were pale yellow, but no leaves fell from either the covered or uncovered plants.

\section{Growth of Plants in Ventilated and Unventilated Chambers of Equal Size}

Series III.--The chambers used in this series were two wooden culture boxes, $20 \times 20 \times 50 \mathrm{~cm}$. Each box had a closely fitting rabbeted door. In each box were placed three 4-inch pots of seedlings of Brassica alba (L.) Boiss. about $4 \mathrm{~cm}$. tall, growing from earth. These boxes were placed in an interior darkroom with a capacity of 
I 2 cubic meters, the air in which was constantly agitated by an electric fan. One box had its door ajar $5 \mathrm{~cm}$., the other was tightly closed. The seedlings in each box numbered 60 . The temperature ranged from $23^{\circ}$ to $25^{\circ}$. The test lasted for 4 days.

During the progress of the experiment, it was necessary to water the plants in the open box twice. The other box was not opened during the 4 days, and the earth was moist at the close of the experiment.

Except for a little more damping off in the closed box (there was some in the open box), no differences could be seen in the two sets of plants at the end of the 4 days. The general thriftiness of the seedlings from the closed box seemed a little greater than that of the seedlings from the open box. Measurements showed no difference in the average height of the two sets of plants.

Series $I V$.-A very satisfactory series of experiments was carried through by employing two large wooden boxes, keeping the air stagnant in one and in movement in the other, the two boxes sitting side by side in a quiet corner of a room with thermostatic control. Each box was $35 \times 60 \times 90 \mathrm{~cm}$., was carefully lined with heavy paper to reduce the exchange of air with the outside, and had a closely fitting cover. To secure agitation of the air in one box, a small blower was set outside, whose inlet and outlet were connected with apertures in the box by short lengths of iron pipe Io $\mathrm{cm}$. wide. It was soon found that the motor of the blower heated the air passing through the blower $1^{\circ}$ to $2^{\circ}$ higher than the air in the other box. The difficulty was overcome by covering a portion of the blower pipe with absorbent paper and causing water to drip on the paper. After some adjustments had been made, the air in the two boxes, as shown by inserted incubator thermometers, kept fairly well the same temperature, never differing in the two boxes more than $0.4^{\circ} \mathrm{C}$. Temperatures during the experiment ranged from $18^{\circ}$ to $21.5^{\circ}$. Observations were made every 2 or 3 days, and the tests lasted from 7 to 19 days.

The following seedlings were tested, growing in pots of earth; the experiments being started as soon as the seedlings had broken through the ground: Zea mais everta Sturtev. I40 plants, Zea mais L. (yellow dent) 4I, Triticum vulgare Vill. 39, Lupinus albus L. I58, Pisum sativum L. 38, Lathyrus odoratus L. I20, Vicia faba M. (Windsor broad) 37 , Ricinus communis L. 12.

The difference in humidity in the two boxes was considerable, 
though much smaller than in the previous comparisons. This reduction of the difference in humidity is followed by the nearly complete disappearance of the greater height attained by plants under the bell jars.

In some of the sets with Zea, seedlings in the box with still air grew thicker and taller; the next week, with a like test, results were exactly reversed. The same reversal occurred with Lathyrus. Lupinus and Vicia gave a little greater average in height and thickness of seedlings in quiet air, Ricinus in moving air, while measurements could detect no difference between the two cultures of Triticum. Lathyrus and both varieties of Zea showed more individuals dying in I 8 days in moving than in quiet air, while, of the other species used, few individuals died and there was no larger percentage of deaths in one box than in the other. Persistent differences in the time of unfolding of leaves in quiet and in moving air could not be established.

Using the same two boxes as with the foregoing seedlings, 6 potted plants of Coleus were employed, 3 in each box. The plants were each about $10 \mathrm{~cm}$. high, each with 4 pairs of mature leaves, and all growing well when they were taken from the greenhouse. To make the moisture conditions more nearly equal in the two boxes, four basins, $20 \mathrm{~cm}$. in diameter, were filled with water and set in the box whose air was to be agitated by the blower.

The 6 plants were kept in the dark boxes for Io days, temperature in the two boxes not differing more than $0.4^{\circ} \mathrm{C}$., and the temperature of the room containing the boxes ranging from $18^{\circ}$ to $21.5^{\circ} \mathrm{C}$. Examination and measurements were made every 2 or 3 days. That the pots in the moving air were still in a drier atmosphere than those in quiet air was shown by the more frequent watering needed by those in moving air. The effects noted in the foregoing cases were still apparent here, though to a very slight degree. At the conclusion of the Io-day period, the 3 plants in moving air showed a total elongation of $13.7 \mathrm{~mm}$., and had dropped 12 leaves. The 3 plants in quiet air showed a total elongation of $14.7 \mathrm{~mm}$., and had dropped Io leaves. Other differences were not apparent.

Series V.-This series of experiments was carried out in two constant temperature dark cabinets of about 12 cubic meters capacity each, in one of which an electric fan was kept in constant motion, while in the other the air was quiet. The temperature for both cabinets was regulated for $23^{\circ}$, with an extreme variation of $I^{\circ} \mathrm{C}$. 
Zea mais (yellow dent) seedlings were set in these cabinets just as the plants were breaking through the earth. In the agitated air were 16 seedlings, in quiet air 17 seedlings. After 6 days, 4 plants in each cabinet had fallen over. The plants in quiet air averaged a little taller than those in moving air. No other differences could be detected. Ten days after the beginning of the test, all plants in moving air had fallen over, and all but one in quiet air had fallen over. The average height of the $I 6$ plants in moving air was $27 \mathrm{~cm}$.; of the 17 in quiet air, $29 \mathrm{~cm}$. Of the plants in moving air, 5 had 3 leaves and I I had 2 leaves each; of those in quiet air, 5 had 3 leaves and I 2 had 2 leaves each. No other differences could be seen between the two sets of plants.

For the sake of comparison with the two foregoing groups of plants, a third set of 15 Zeas was run at the same time under small zinc cylinders in one of the cabinets. At the end of the 6-day period, I I of the I 5 had fallen over, compared with 4 in each of the other sets. At the end of the Io days, all 15 plants had fallen over. The average height of these was $37 \mathrm{~cm}$. compared with 27 and $29 \mathrm{~cm}$. respectively in the other two groups; and of the plants under the zinc covers, Io had 3 leaves and 5 had 2 leaves, there being thus twice as many with 3 leaves each as in each of the other two groups. These comparative results bring out strongly the effect of excessive moisture induced by a small confined space.

None of the falling of the plants noted above was due to damping off.

Fagopyrum esculentum seedlings were placed in the dark cabinets, 4 pots in each, with Io seedlings in each pot, seedlings grown in the greenhouse to a height of 2 to $5 \mathrm{~cm}$. The temperature was $23^{\circ} \mathrm{C}$. in each cabinet, the air agitated in one, quiet in the other.

After 6 days, 29 of the 40 seedlings in quiet air and 33 of the 40 in moving air had fallen over. Thus there were 7 erect in moving air and I I erect in quiet air. The number of dead seedlings was about the same in each group. As the experiment progressed during the 6 days, no certain difference in general behavior could be observed. The small difference noted above is probably not significant; another test might reverse relations.

Lupinus albus seeds were planted in whitewood (Liriodendron) sawdust, from which 38 seedlings grew in one pot and 46 in the other. When the first seedlings appeared above the sawdust, the pots were removed to the two dark cabinets, one pot in moving, the other in 
quiet air. The growth and general behavior were followed for the ensuing Io days, but no differences could be detected. The seedlings began falling over from weakness 7 days after they had been placed in the cabinets. At this time, the largest seedlings in each pot were I6 to I9 $\mathrm{cm}$. high. Of the 38 seedlings in moving air, 9 showed primary leaves emerging between the half-open cotyledons; while among the 46 seedlings in quiet air, I I showed primary leaves emerging between the opening cotyledons. At the end of Io days in the dark cabinets, the most of the seedlings in each pot had fallen over. None were dead, and there was no damping off.

The temperature during the experiment had ranged from $22^{\circ}$ to $23^{\circ}$.

Brassica alba seedlings, to the number of 72 in moving air and 63 in quiet air, were grown in pots of earth, the preparations being placed in the two dark cabinets before the seedlings had broken through the ground. The plants grew in the two cabinets without showing noticeable differences, except that the cotyledons opened a little earlier in the quiet air; on the fifth day of the experiment, I 2 seedlings in moving air and 16 in quiet air showed expanded cotyledons. On the sixth day, all but 8 seedlings in moving air, and all but 19 in quiet air had fallen over. The Io longest hypocotyls in moving air averaged I $5 \mathrm{~cm}$.; in quiet air, the ro longest averaged $14.5 \mathrm{~cm}$. There were no cases of damping off.

The temperature had ranged for the 6 days from $22^{\circ}$ to $23^{\circ}$, and kept the same in both cabinets.

Cucurbita pepo L., with seedlings to the number of 63 , gave the same general result as the foregoing species. Differences in behavior in moving and in quiet air in the cabinets could not be discerned.

\section{Sensitive Reactions in Ventilated and Unventilated Chambers}

Besides the criteria of the amount of growth, unfolding of leaves, and the vital period, used in the foregoing pages to estimate the effect of ventilation, it would be worth while to employ sensitive reactions also as a criterion. For this purpose geotropism and heliotropism have been studied with the plants in two small culture boxes, the one ventilated, the other closed against the exchange of air. These boxes were of wood $20 \times 20 \times 50 \mathrm{~cm}$., with a closely fitting rabbeted door, and an aperture in one end $5 \times 10 \mathrm{~cm}$. The boxes stood side by side in a darkroom in which an electric fan was kept in constant motion. 
The temperature of the darkroom was kept at $24^{\circ}$ to $25^{\circ} \mathrm{C}$. The aperture in the end of one box was tightly closed by a glass plate held in position by a border of heavy paper pasted to the glass and the wall of the box. The fan was placed so that a gentle current of air flowed obliquely against the aperture end of each box.

Geotropism.-Three 4-inch pots of seedlings of Brassica alba, 20 seedlings in each pot, the seedlings raised in the same darkroom as that in which the later experiment was made, and having a height above the earth of 2.5 to $5 \mathrm{~cm}$., were placed in each culture box in the erect position, and so left for 18 hours. Without opening, the boxes were now turned so that the included pots were brought into the horizontal position, the pots having previously been secured to prevent rolling.

Observation at the end of two and one half hours showed in the ventilated box 50 of the 60 plants with geotropic angles of $15^{\circ}$ to $90^{\circ}$; in the closed box, 48 of the 60 showed corresponding curves. The angles seemed to average a few degrees more in the closed box, but this was only an estimate. No accurate measurements were made.

Six and one half hours after turning the plants to the horizontal position, all the seedlings in the ventilated box and all but one in the closed box showed negative geotropic curves of $20^{\circ}$ to $90^{\circ}$. A greater average angle for either set of plants could not be determined.

Heliotropism.-Six 4-inch pots of seedlings of Brassica alba were raised in the greenhouse to a height of 2 to $3 \mathrm{~cm}$. above the earth, then transferred to the dark cabinet last used, and 3 pots enclosed within the closed culture box used in the preceding experiment, while the other 3 were placed in the ventilated culture box. In this condition the 6 pots remained in the dark for 18 hours, the electric fan keeping the air of the cabinet in circulation. In order that the plants in both culture boxes might have the same amount of light in the subsequent stimulation, a glass plate, like that used in closing one of the culture boxes, was placed inside the other box and $4 \mathrm{~cm}$. distant from the aperture, so that all light which reached the plants had to pass through similar glass in each box, but the current of air could enter one box and not the other.

A single tungsten lamp of 25 watts was used for both boxes, the boxes being set with their long axes pointing toward the lamp. The middle of the pots in each box were respectively 25,38 , and $51 \mathrm{~cm}$. distant from the lamp. The temperature was $24^{\circ} \mathrm{C}$. 
After the preparations had been in the dark for 18 hours, the light was turned on. Observation after one hour showed the seedlings in each box in the pot nearest the light responding with positive curves. The responses seemed about equal in the two boxes. There were a total of 62 seedlings in the ventilated box, and $7 \mathrm{I}$ in the unventilated box. Two hours after the light was turned on, the pots in the ventilated box, arranged in the order of their nearness to the light, showed respectively 95 percent, 73 percent, and $2 \mathrm{I}$ percent of the seedlings with positive angles of $20^{\circ}$ to $60^{\circ}$. Those in the unventilated box, in the same order, showed 100 percent, 48 percent and 45 percent with positive angles of $20^{\circ}$ to $60^{\circ}$. The average angle seemed about the same in both boxes.

Six hours after the illumination began, in the ventilated box I2 of the 62 seedlings were still erect. In the unventilated box, 7 of the 7 I seedlings were still erect. This difference is not significant. The experiment ends with no evidence tending to show that confinement in a small unventilated chamber retards heliotropic response.

The foregoing test was repeated with 3 pots of seedlings of Brassica alba, and 3 pots of seedlings of Fagopyrum esculentum in the ventilated box, and the same number of pots of each species in the unventilated box. At the same time 3 pots of each species were put into an adjoining cabinet of equal size (I2 cubic meters), where the temperature remained the same as in the first cabinet, and where the air was quiet. The 6 pots last mentioned were not put into a culture box.

Early observation for incipient heliotropic curvature was not made; but, after the illumination had continued for 12 hours, all plants had responded, and no differences in the behavior of the 3 groups of seedlings could be detected.

\section{Summary AND Conclusions}

The work narrated in the present paper was undertaken to determine whether plants behave as well in quiet air in confined chambers as in moving air or in ventilated chambers. The question is of practical importance in experimental work chiefly in the growth of plants in dark boxes and darkrooms. The growth of plants in stagnant air in the light involves several other conditions, and no experiments in the light were undertaken.

The material employed was mostly seedlings, of which about 2,000 have been used, belonging to 12 species. Larger plants belonging to 3 species were also used. 
The chambers for securing stagnant air have varied in size from bell jars of about 6 liters capacity to darkrooms of 12 cubic meters. While one set of plants was growing in the chambers in quiet air, another control set was always growing in a chamber of equal or larger size whose air was stirred constantly with a fan or with a blower.

The criteria for comparing behavior in quiet and moving air were duration of vital period in the dark, size of seedlings, size and number of leaves formed, general vigor of the plants, phenomena of geotropic and heliotropic reaction.

It may be said in a word that no ill effects manifested themselves as due to confining plants in stagnant air in small or in large chambers. The greatest objection to small chambers, as bell jars, comes from the growth of injurious fungi on the plants, due to the excessive moisture. This ill effect mostly disappears when the plants are kept in a larger chamber, or when care is taken to keep the uppermost stratum of the soil free from excessive moisture.

With but few exceptional cases, the cultures have shown that in small chambers, like bell jars, seedlings grow taller, produce larger and more numerous leaves, and become exhausted so that they fall over a day or two earlier $\left(\right.$ at $20^{\circ}$ ) than in a larger ventilated chamber. These differences were pronounced when comparing plants grown under bell jars with similar plants grown in moving air, the moving air coming either from the outside or being circulated within the darkroom. The differences were much less when the chamber with stagnant air was increased to a fifth of a cubic meter, and disappeared in most cases when the two chambers for comparison were 12 cubic meters each, the one with stagnant, the other with circulating air.

This larger and more rapid growth in very moist air is no new discovery. It has been cited by Wiesner ${ }^{3}$ and by Eberhardt. ${ }^{4}$

Not only is ventilation of no effect in producing better seedlings in a small or large chamber in the dark, but it has no visible effect on the sensitive reactions of geotropism and heliotropism.

Botanical LaBoratory,

UNIVERSITY OF MiChigAN.

${ }^{3}$ Wiesner. Formänderungen von Pflanzen bei Cultur im absolut feuchten Raume und im Dunkeln. Ber. Deutsch. Bot. Ges. 9: 46. I89I.

4 Eberhardt. Action de l'air humide sur les végétaux. Compt. Rend. Acad. Sci. (Paris) I31: I93. I900. 